\title{
Changes in the Brain's Intrinsic Organization in the Resting State with Real-Time fMRI Neurofeedback Training of Posterior Cingulate Cortex Activity
}

\author{
Yubao Wang1, Jipeng Zhang², Gaoyan Zhang³, Li Yao1,2, Zhiying Long1* \\ ${ }^{1}$ State Key Laboratory of Cognitive Neuroscience and Learning \& IDG/McGovern Institute for Brain Research, \\ Beijing Normal University, Beijing, China \\ ${ }^{2}$ College of Information Science and Technology, Beijing Normal University, Beijing, China \\ ${ }^{3}$ School of Computer Science and Technology, Tianjin Key Laboratory of Cognitive Computing and Application, \\ Tianjin University, Tianjin, China \\ Email: ^friskying@163.com
}

How to cite this paper: Wang, Y.B., Zhang, J.P., Zhang, G.Y., Yao, L. and Long, Z.Y. (2017) Changes in the Brain's Intrinsic Organization in the Resting State with Real-Time fMRI Neurofeedback Training of Posterior Cingulate Cortex Activity. Journal of Behavioral and Brain Science, 7 , 655-673.

https://doi.org/10.4236/jbbs.2017.713044

Received: September 6, 2017

Accepted: December 4, 2017

Published: December 8, 2017

Copyright (c) 2017 by authors and Scientific Research Publishing Inc. This work is licensed under the Creative Commons Attribution International License (CC BY 4.0).

http://creativecommons.org/licenses/by/4.0/

\section{c) (i) Open Access}

\begin{abstract}
Real-time functional magnetic resonance imaging (rtfMRI) technology has been widely used to train subjects to actively regulate the activity of specific brain regions. Although many previous studies have demonstrated that neurofeedback training alters the functional connectivity between brain regions in the task state and resting state, it is unclear how the regulation of the key hub of the default mode network (DMN) affects the topological properties of the resting-state brain network. The current study aimed to investigate what topological changes would occur in the large-scale intrinsic organization of the resting state after the real-time down-regulation of the posterior cingulate cortex (PCC). The results indicated that the down-regulation of the PCC in the DMN reduced the functional connectivity of the PCC with the nodes outside of the DMN and reduced functional connectivity between the superior medial frontal gyrus (SFGmed) and parahippocampal gyrus (PHG) in the experimental group. Moreover, the nodal graph properties of the SFGmed were reduced, while that of the PHG showed the opposite alteration after the down-regulation of the PCC. These findings possibly suggest that the regulation of the key hub of the DMN, the PCC, mainly changed the information transfer of the SFGmed and PHG.
\end{abstract}

\section{Keywords}

Real-Time fMRI, Neurofeedback, Default Mode Network, Posterior Cingulate Cortex, Graph Theory 


\section{Introduction}

Functional magnetic resonance imaging (fMRI) is a noninvasive technique that can be used to assess brain function by measuring blood-oxygen-level-dependent signal changes [1]. By far, task-based and resting-state fMRI are the two most popular paradigms to investigate brain function. Task-based fMRI paradigms are generally used to reveal differentiated involvement of brain regions by comparing the experimental condition with the "rest" condition. However, it was noted that the brain is not idle at "rest" but rather produces spontaneous intrinsicactivity that is highly correlated between multiple brain regions [2]. Especially in recent years, resting-state fMRI (RS-fMRI) has attracted an increasing amount of attention to investigate synchronous activity between brain regions and identify resting-state networks [3].

Resting-state functional connectivity (RSFC) research has revealed a number of consistent resting networks that represent specific patterns of synchronous activity from healthy subjects [4]. Moreover, the RSFC in the human brain is dynamic. Previous studies have demonstrated that the RSFC can be altered by development, aging [5], or neurologic and psychiatric brain disorders that include schizophrenia, Alzheimer's disease, dementia and depression [6]. Furthermore, some studies have reported that the RSFC can be modulated by offline learning-related training [7] [8]. These studies suggest that the RSFC is alterable and can be affected by appropriate training. Thus, the dynamic characteristics of the RSFC are important for us to explore the functional stability and flexibility of the brain.

Recently, real-time functional magnetic resonance imaging (rtfMRI) technology has been used to train subjects to actively control their brain activity [9]. The self-regulation of brain activity can lead to changes in functional connectivity during the task state. Several studies have demonstrated that functional connectivity of the task fMRI can be altered by neurofeedback training through real-time modulation of the activity of the premotor area, visual cortex, amygdala, insular cortex and primary auditory cortex [10], etc. Moreover, rtfMR Ineurofeedback training can also change the functional connectivity during the resting state. Hampson et al. [11] reported that the RSFC of the supplementary motor area with the left striatum and right thalamus during the resting state was significantly reduced after real-time modulation of the activity of the supplementary motor area. Scheinost et al. [12] found that orbitofrontal cortex neurofeedback produced lasting changes in the RSFC in limbic circuitry and the dorsolateral prefrontal cortex. Yuan et al. [13] observed that the RSFC of the amygdala with the right parahippocampalgyrus, right superior temporal gyrus, bilateral middlefrontal gyrus and right lingual gyrus was significantly changed through real-time modulation of the activity of amygdala. Megumi et al. [14] reported that the RSFC of the regions of the default mode network (DMN) with the regions of the motor/visuospatial network was significantly increased by real-time regulation of connectivity between two regions. Moreover, it was 
demonstrated that the neurofeeback training on connectivity between two regions could induce long-lasting changes in intrinsic functional network [14]. Although these studies suggest that neurofeedback training induces changes in RSFC, it is not clear how the intrinsic organization of the brain in the resting state changes after neurofeedback training. Especially, it is unknown how neurofeedback of the key hub of the DMN alters the intrinsic organization of the brain in the resting state.

In our previous study, the down-regulation of the activity of the PCC was demonstrated to alter the activity of the DMN in the resting state using the rtfMRI technique and the Independent Component Analysis (ICA) method [15]. The ICA method is generally used to reveal the pattern of functionally connected brain networks but cannot reveal how the nodes within a brain network interact with each other. Thus, it is unknown what changes would occur in the intrinsic organizational mechanism of the brain underlying the relevant networks through the down-regulation of the activity in the PCC. Based on our previous study, this study aimed to further investigate the topological alterations of the intrinsic organization of the brain during the resting state after real-time neurofeedback training in terms of: 1) the small-world configuration and 2) the global DMN topography and nodal properties. In contrast to ICA, the graph-based network analyses can not only provide the visualization of the overall connectivity pattern among all of the elements of the brain regions but also quantitatively characterize the global organization [16]. Therefore, the graph theory method was used in this study to identify changes in the topological properties of the brain functional network during the resting state after real-time neurofeedback training. The RS-fMRI data of 16 subjects in the experimental group before and after real-time neurofeedback training were acquired. Moreover, the RS-fMRI data of 16 subjects in the control group that underwent the same task but without neurofeedback during the training were also collected. We hypothesized that the regulation of the PCC could result in the topological alteration of the key nodes and the alteration of connectivity between the key nodes of the DMN.

\section{Material and Methods}

The data used in this study were collected in our previous study [15]. For readability, the main information about the subjects, experimental procedure and scanning parameters are repeated here.

\subsection{Participants}

Thirty-two healthy right-handed individuals with normal vision participated in the experiment. The experimental group included eight females and eight males (age: $21.00 \pm 2.00$ years) and the control group included eight females and eight males (age: $21.60 \pm 2.00$ years). The two groups had no significant difference in age $(p=0.18)$. All participants agreed to sign informed consent before scanning. 


\subsection{Imaging Parameters}

The brain scans were performed at the MRI Center of Beijing Normal University using a 3.0-T Siemens whole-body MRI scanner. A single-shot T2*-weighted gradient-echo, EPI sequence was used for functional imaging acquisition with the following parameters: TR/TE/flip angle $=2000 \mathrm{~ms} / 30 \mathrm{~ms} / 90^{\circ}$, matrix $=64 \times$ 64 , in-plane resolution $=3.125 \times 3.125 \mathrm{~mm}^{2}$, slice $=33$, slice thickness $=4 \mathrm{~mm}$, and slice gap $=0.6 \mathrm{~mm}$.

\subsection{Experimental Procedure}

The whole experiment included a task familiarization exercise, a pre-training resting run, a region of interest (ROI) localizer run, two training runs, a post-training resting run and a questionnaire interview outside of the scanner. Our previous study demonstrated that the task of moving and imaging the right fingers according to the sequence 4-2-3-1-3-4-2 can make the region in PCC deactivated stably [17]. In order to identify the reliable PCC and down-regulate PCC effectively, the same finger tapping task was used to locate the PCC ROI and the finger imagination strategy was used during the neurofeedback training runs.

\subsubsection{Task Familiarization Exercise}

In the task familiarization exercise, all subjects were told that the four fingers of their right hand from their index to little finger represented one, two, three, and four, respectively. Then, they were required to perform a right-hand finger movement for 30-s according to the sequence 1-2-3-4 with a metronome set to 4 $\mathrm{Hz}$ and imagine the movement of the right fingers for $30 \mathrm{~s}$ according to the sequence 1-2-3-4 without a metronome.

\subsubsection{Pre-Training Resting Run}

All subjects were instructed to remain still for $10 \mathrm{~min}$ with eyesclosed in this run.

\subsubsection{ROI Localizer Run}

The 3.5-min localizer run consisted of three 30-s task blocks alternating with four 30-s rest blocks. During the task blocks, subjects performed the right-hand finger movement according to a new sequence4-2-3-1-3-4-2 at $4 \mathrm{~Hz}$ without a metronome. The ROI was selected as a rectangular zone in one slice centered on the area of deactivation of the PCC. The volume and location of the ROI varied across subjects (mean volume: $15.90 \pm 3.20$; mean slice: $13.20 \pm 0.80$ ). The volume represented the number of voxels in the ROI.

\subsubsection{Two Neurofeedback Training Runs}

Each training run lasted $782 \mathrm{~s}$ and consisted of eight 46-s task blocks alternating with nine 46-s rest blocks. The neurofeedback presented to the subjects was a continuously updated time course averaged across the voxels in the ROI. All subjects in the experimental group were required to lower the activity in the ROI during the task blocks and maximizing the difference between the activity of the 
ROI during the rest and task blocks. Apart from imagining, subjects were instructed to imagine the movement of the right fingers according to the sequence 4-2-3-1-3-4-2 with varied speed, strength and the mode of the movement during the imagery task. During the rest blocks, subjects were required to rest and not recall anything about the regulation. The subjects in the control group were required to imagine movements of the right fingers according to the sequence 4-2-3-1-3-4-2 without neurofeedback signal during the task blocks.

\subsubsection{Post-Training Resting Run}

All subjects were asked to stay relaxed with eyes closed during the 10-min post-training resting run.

\subsubsection{Questionnaire Interview}

After the scan, a questionnaire was filled out by each subject. The questionnaire mainly addressed whether the subjects performed the tasks according to the experimenter's instruction, the detailed strategies they used to regulate the activity and any difficulties they encountered during the experiment.

\subsection{Data Analyses}

For the pre-training and post-training resting data of each subject, the preprocessing, brain network construction and the calculation of global network parameters and regional nodal parameters were performed using graph theoretical network analysis software (GRETNA, http://www.nitrc.org/projects/gretna).

\subsubsection{Preprocessing}

The preprocessing steps included removal of the first 10 volumes, slice timing correction, head movement correction, spatial normalization (EPI template provided by the Montreal Neurological Institute, MNI, with a final resolution of $3 \times$ $3 \times 3 \mathrm{~mm})$, removal of linear trend, temporal band-pass filtering $(0.01-0.08 \mathrm{~Hz})$ and nuisance signal regression ( 6 head motion parameters, the cerebrospinal fluid signal and the white matter signals). Two subjects in the experimental group were eliminated because the target ROI could not be defined. In addition, two subjects in the experimental group and four in the control group were further removed from the analysis because the translation of head movement was larger than one voxel during training. As a result, a total of 24 subjects consisting of 12 in the experimental group and 12 in the control group underwent the subsequent brain network analysis.

\subsubsection{Construction of Large-Scale Brain Functional Networks}

Each participant's brain was parceled into 90 cortical and subcortical regions using the AAL atlas. Then, the time series of each ROI was acquired by averaging the signals of all voxels within each ROI region. Pearson's correlation coefficients of time series between any pair of brain regions were calculated, and a Fisher's r-to-z transformation [18] was applied to improve the normality of the correlation coefficients. For each resting run of each subject, a temporal correla- 
tion matrix $(90 \times 90)$ was obtained. A sparsity threshold was used to threshold each correlation matrix into a binarized matrix to ensure that the brain networks corresponding to each subject had the same number of edges. Sparsity is defined as the ratio of the number of actual edges divided by the maximum possible number of edges in a network. The positive value of the Fisher Z-score was employed for sparsity thresholding. We set a series of sparsity threshold values in a range of $0.1-0.5$ at intervals of 0.01 to allow prominent small-world properties in brain networks to be observed [19]. The undirected binarized graphs of the brain were obtained with the nodes representing brainregions and the edges representing functional relationships between brain regions.

\subsubsection{Network Analysis}

The network analysis, including the global network parameters and regional nodal parameters, was performed in the large-scale brain functional networks.

Global network parameters. Graph theory has been widely used to quantitatively characterize the brain functional networks [20]. In this study, six global network parameters that included four small-world parameters (clustering coefficient $C_{p}$, characteristic path length $L_{p}$, normalized clustering coefficient $\gamma$, and normalized shortest path length $\lambda$ ) and two efficiency parameters (global efficiency $E_{\text {glob }}$ and local efficiency $E_{l o c}$ ) were used to characterize the global topological organization of brain networks. Their formula definitions in a graph $G$ with $N$ nodes are listed below [21] [22],

$$
C_{p}=\frac{1}{N} \sum_{i \in G} \frac{K_{i}}{D_{\text {nod }}(i)\left(D_{\text {nod }}(i)-1\right) / 2}
$$

where $N$ is the number of all nodes of a network $G, D_{\text {nod }}(i)$ is the degree of node $i$, and $K_{i}$ is the number of edges in the subgraph $G_{i}$ that consists of the neighbors of node $i$. $C_{p}$ measures the local cliquishness of a network $G$,

$$
L_{p}=\frac{1}{\frac{1}{N(N-1)}\left(\sum_{j \neq i \in G} \frac{1}{L_{i j}}\right)}
$$

where $L_{i j}$ denotes the shortest path length between nodes $i$ and $j . L_{p}$ measures the overall routing efficiency of a network $G$.

$$
E_{g l o b}=\frac{1}{N(N-1)} \sum_{j \neq i \in G} \frac{1}{L_{i j}}
$$

$E_{\text {glob }}$ measures the extent of information propagation through the whole network.

$$
E_{\text {loc }}=\frac{1}{N} \sum_{i \in G} E_{\text {glob }}(i)
$$

$E_{l o c}$ measures the capability of parallel information transfer in the local scope of a network.

To examine the small-world properties, the normalized clustering coefficient $\gamma=C_{p}^{\text {real }} / C_{p}^{\text {rand }}$ and the normalized shortest pathlength $\lambda=L_{p}^{\text {real }} / L_{p}^{\text {rand }}$ were 
computed [23]. $C_{p}^{\text {real }}$ and $L_{p}^{\text {real }}$ are the clustering coefficient and the shortest pathlength of real networks, respectively. $C_{p}^{\text {rand }}$ and $L_{p}^{\text {rand }}$ represent the mean indices derived from matched random networks (100matched random networks that preserved the same number of nodes, edges, and degree distribution as the real networks were selected) [24]. Typically, a small-world network should meet the following criteria: $\gamma>1$ and $\lambda \approx 1$ [23], or $\sigma=\gamma / \lambda>1$ [25].

Regional nodal parameters of DMN nodes. Among the various resting networks, the DMN is a prominent one that reflects a default state of neuronal activity of the human brain. Moreover, the PCC that was down-regulated during neurofeedback training was a key hub in the DMN [26]. Thus, this study focused on investigating the regional nodal parameters of the DMN nodes. In the present study, three nodal parameters that included degree $\left(D_{\text {nod }}\right)$, nodal efficiency $\left(E_{n o d}\right)$, and betweenness centrality $(\mathrm{BC})$ were used to examine the regional characteristics of brain network. The following are their formula definitions in a graph $G$ with $N$ nodes [27] [28]:

$$
D_{\text {nod }}(i)=\sum_{j \neq i \in G} e_{i j}
$$

where $e_{i j}$ is the $(i, j)$ th element in the formerly obtained binarized correlation matrix. $D_{\text {nod }}(i)$ measures the connectivity of node $i$ with the rest of the nodes in a network.

$$
E_{\text {nod }}(i)=\frac{1}{N-1} \sum_{j \neq i \in G} \frac{1}{L_{i j}}
$$

$E_{\text {nod }}(i)$ measures the ability of information transmission of node $i$ in the network.

$$
B C(i)=\sum_{j \neq i \neq k \in G} \frac{\delta_{j k}(i)}{\delta_{j k}}
$$

$\delta_{j k}$ is the number of the shortest paths from node $j$ to node $k$, and $\delta_{j k}(i)$ is the number of the shortest paths from node $j$ to node $k$ that passes through node $i$ within the network $G$. BC(i) measures the influence of node i over information flow between other nodes in the brain network. Moreover, the nodal characteristics of the brain networks measure the extent to which a given node connects to all other nodes of a network, which may indicate the importance of special brain areas in the brain network [27].

\subsubsection{Extraction of the Global DMN Topography}

In the present work, the DMN regions were determined from the AAL-at-asprimarily according to the coordinates of the peak foci of all the "task-negative" regions [29]. The ROIs in the AAL-atlas which covered the coordinates or the most adjacent ones were selected. Two coordinates $(-3,39,-2)$ and $(1,54,21)$ were close to the inter-hemisphere locations between the left and right homogeneous regions, so the corresponding bilateral AAL-atlas regions were also selected. Last, the peak coordinate $(-2,-36,37)$ is near the boundary between cingulum_Mid_L and cingulum_Post_L, but the great part ofthe left PCC in Fox et al. (2005) is involved in cingulum_Post_L, so we selected cingulum_Post_L as 
the corresponding left PCC. Totally fourteen regions were selected as the AAL-based DMN components (see Table 1). For each resting run of each subject, a correlation sub-matrix representing the global DMN topography was extracted from the $90 \times 90$ global correlation matrix with the Fisher's r-to-z transformation. The global DMN topography included the pairwise functional connectivity between the fourteen DMN regions (the intra-DMN functional connectivity) and that between the DMN regions and non-DMN regions (the extra-DMN functional connectivity).

\subsubsection{Statistical Analysis}

For each of the global network parameters and regional nodal parameters over the sparsity range of $0.1-0.5$, a two-way repeated-measures analysis of variance (ANOVA) using group (experimental group and control group) as a betweensubject factor and state (pre-training and post-training) as a repeated-measures factor was conducted in SPSS 20.0. Moreover, the same two-way repeated-measures ANOVA was performed on each functional connectivity of the global DMN topography. If any parameter showed a significant interaction effect $(p<$ $0.05)$, tests of simple effect were further carried out and were corrected by the false discovery rate (FDR) method [30] to examine the differences between the pre-training and post-training resting states in each group.

\section{Result}

\subsection{Stability of the Small-World and Global Network Parameters under the Four Conditions}

Figure 1 shows the small-world properties of the functional organization of the brain in the pre-training and post-training resting states for both groups. It can be seen that over the sparsity range of $0.1-0.5, \gamma$ was larger than 1 , and $\lambda$ ap-

Table 1. DMN components (Fox et al., 2005) defined in the AAL-atlas.

\begin{tabular}{ccc}
\hline Regions of interest in the AAL & Talairach coordinates $(\mathrm{x}, \mathrm{y}, \mathrm{z})$ & Common names \\
\hline Frontal_Sup_L & $(-14,38,52)$ & Superior frontal gyrus, \\
dorsolateral \\
Frontal_Sup_R & $(17,37,52)$ & Superior frontal \\
gyrus, medial \\
Frontal_Sup_Medial_L & $(-5,49,31)$ & Anterior cingulate \\
Frontal_Sup_Medial_R & $(9,50,30)$ & and paracingulate gyri \\
Cingulum_Ant_L & $(-3,39,-2)$ & Posterior \\
Cingulum_Ant_R & $(8,37,15)$ & cingulate gyrus \\
Cingulum_Post_L & $(-2,-36,37)$ & Parahippocampal \\
Cingulum_Post_R & $(3,-51,8)$ & gyrus \\
ParaHippocampal_L & $(-22,-26,-16)$ & Angular \\
ParaHippocampal_R & $(25,-26,-14)$ & \\
Angular_L & $(-47,-67,36)$ & Middle temporal gyrus \\
Angular_R & $(53,-67,36)$ & Inferior temporal gyrus \\
Temporal_Mid_R & $(65,-17,-15)$ & \\
Temporal_Inf_L & $(-61,-33,-15)$ & \\
\hline
\end{tabular}




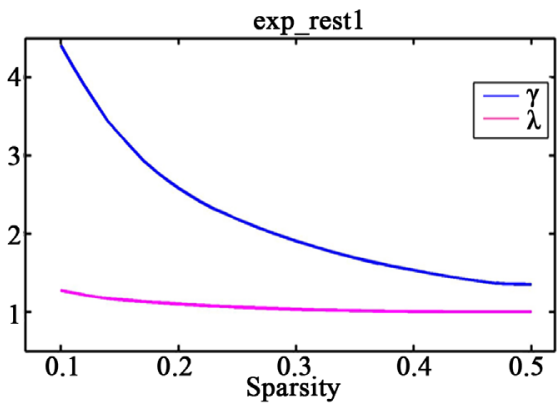

(a)

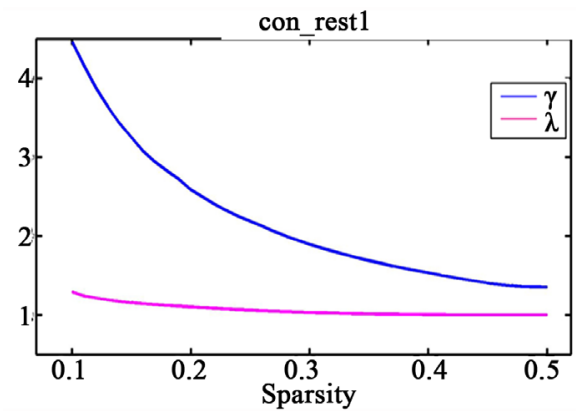

(c)

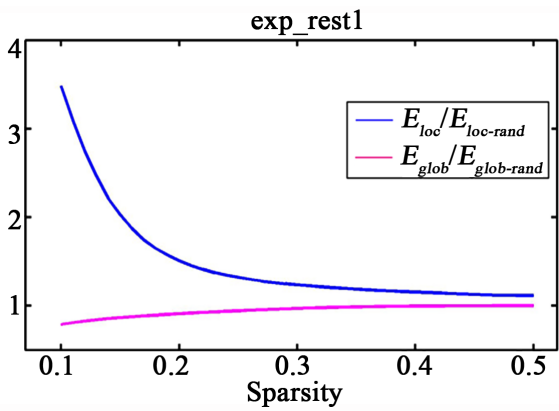

(e)

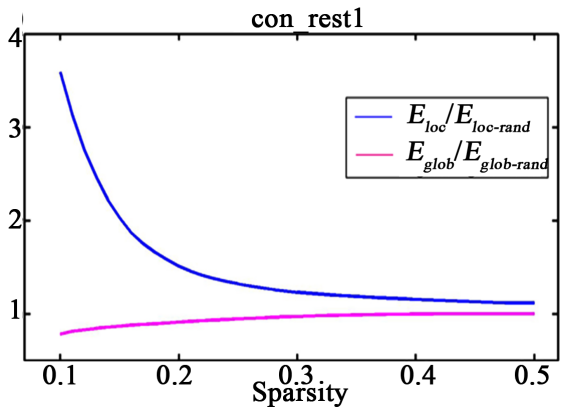

(g)

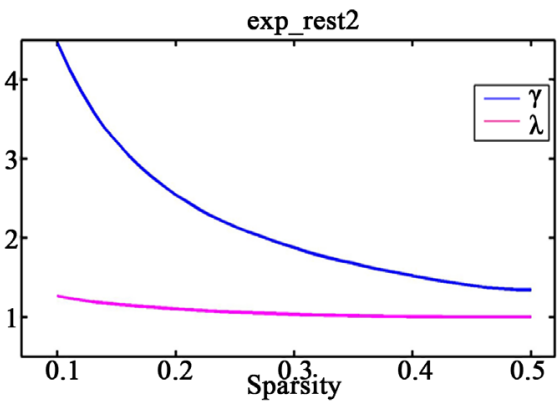

(b)

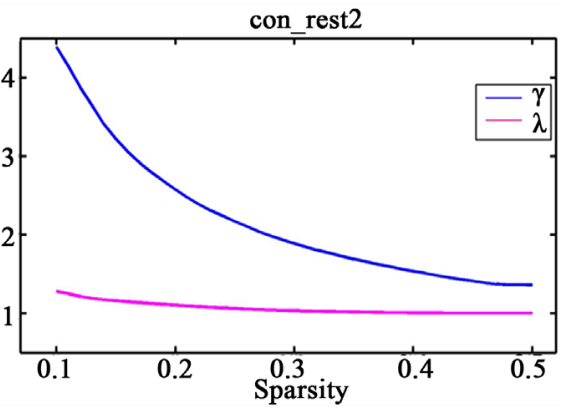

(d)

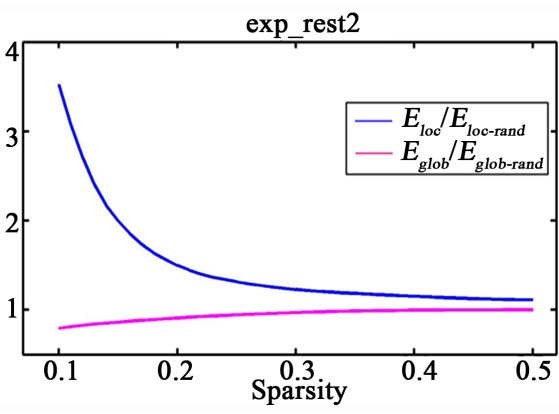

(f)

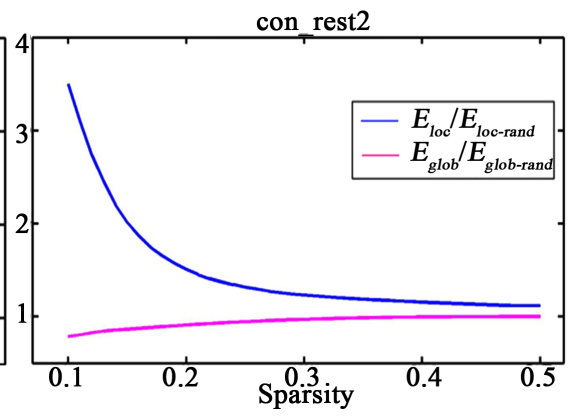

(h)

Figure 1. Small-world properties of the functional organization of the brain in the pre-training and post-training resting states for both groups. ((a)-(d)) Changes in the $\gamma$ and $\lambda$ with the increasing of sparsity thresholds for the pre-training (a) and post-training (b) resting runs of the experimental group and the pre-training (c) and post-training (d) resting runs of the control group; ((e)-(h)) Changes in the $E_{\text {loc }} / E_{\text {loc-rand }}$ and $E_{\text {glob }} / E_{\text {glob-rand }}$ with the increasing of sparsity thresholds for the pre-training (e) and post-training (f) resting runs of the experimental group and the pre-training ( $g$ ) and post-training $(\mathrm{h})$ resting runs of the control group. 
proached 1 for the pre-training and post-training resting states of each group (see Figures 1(a)-(d)). According to Watts and Strogatz (1998), all four sets of networks exhibited small-worldness $(\gamma>1$ and $\lambda \approx 1$ ) in the range of $0.1 \leq$ sparsity $\leq 0.5$. From the efficiency perspective, the local efficiencies of these networks were larger than the matched random networks $\left(E_{\text {loc }} / E_{\text {loc-rand }}>1\right)$, whereas the global efficiencies of these networks approached that of the matched random networks ( $E_{\text {glob }} / E_{\text {glob-rand }} \approx 1$ ) (see Figure $1(\mathrm{e})$-(h)). Thus, these results demonstrated the small-world configurations in the functional network of the brain under the four conditions ( 2 groups $\times 2$ states). Moreover, two-way repeatedmeasures ANOVA did not reveal a significant interaction between group and state on any of the six global parameters of the brain functional network ( $p>$ $0.1)$.

\subsection{Results of the DMN Nodal Graph Properties}

The degree, nodal efficiency and betweenness centrality of the SFGmed. L/R, the betweenness centrality of the cingulum_Post_L (PCC.L), the nodal efficiency and betweenness centrality of the cingulum_Post_R (PCC.R) and the betweenness centrality of the PHG.R showed a significant interaction effect $(p<0.05)$ within some ranges of sparsity levels. Figure 2 and Figure 3 show the variation

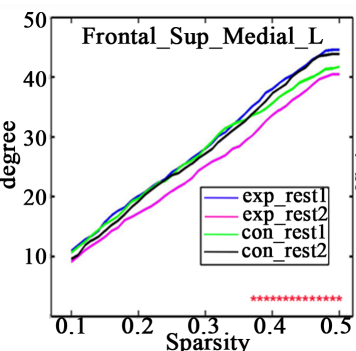

(a)

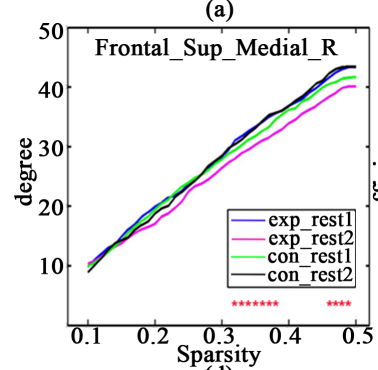

(d)

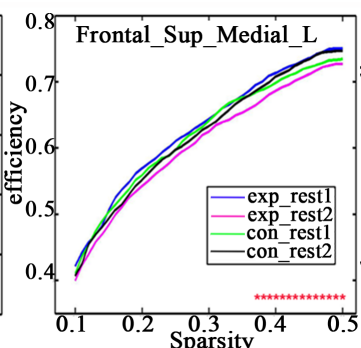

(b)

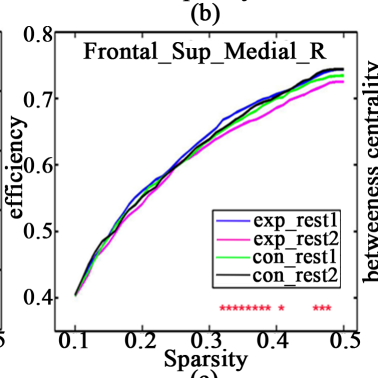

(e)

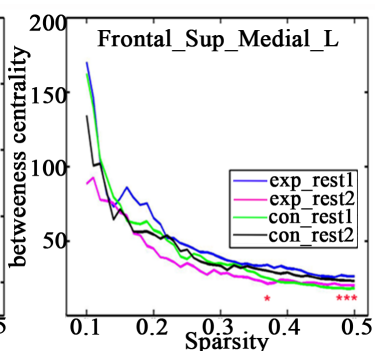

(c)

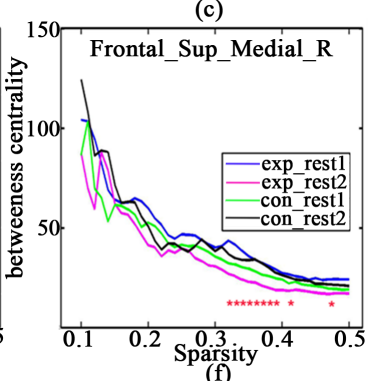

Figure 2. The variation of the nodal parameters that show significant interaction effect with the sparsity threshold for SFGmed. ((a)-(c)) Changes of degree (a); efficiency (b) and betweenness centrality (c) of the SFGmed.L during the pre-training and post-training resting runs for both groups. ((d)-(f)) Changes of degree (d); efficiency (e) and betweenness centrality (f) of the SFGmed.R during the pre-training and post-training resting runs for both groups. Exp_restl represents the pre-training resting run of the experimental group. Exp_rest2 represents the post-training resting run of the experimental group. Con_rest1 represents the pre-training resting run of the control group. Con_rest2 represents the post-training resting run of the control group. Red asterisks indicate the significant difference between the pre-training and post-training resting runs of the experimental group $\left({ }^{\star} \mathrm{FDR}\right.$-corrected $\left.p<0.05\right)$. 


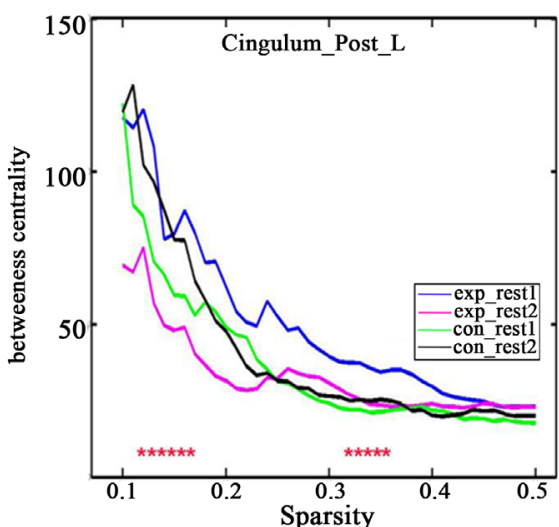

(a)

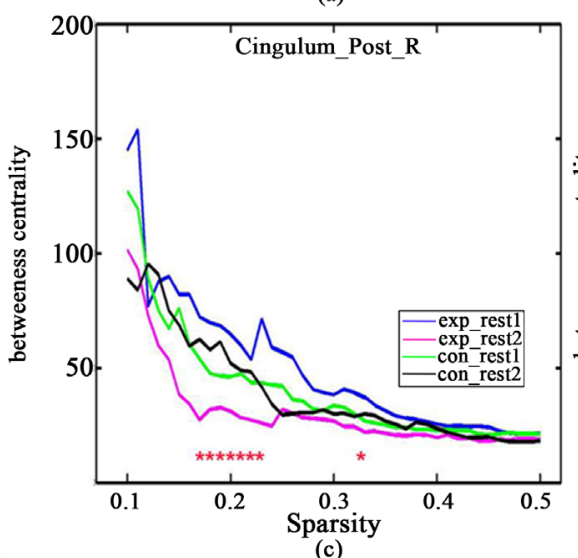

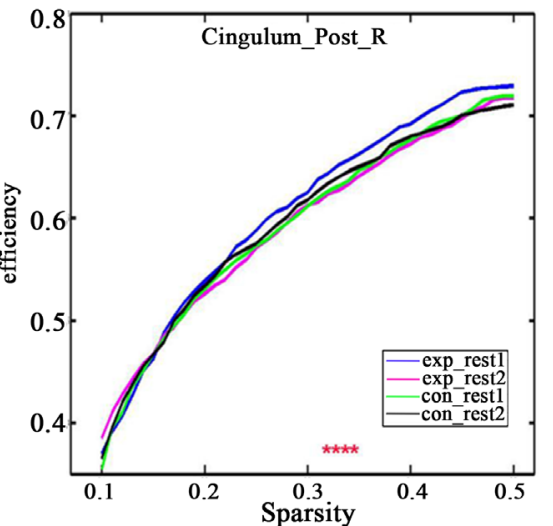

(b)

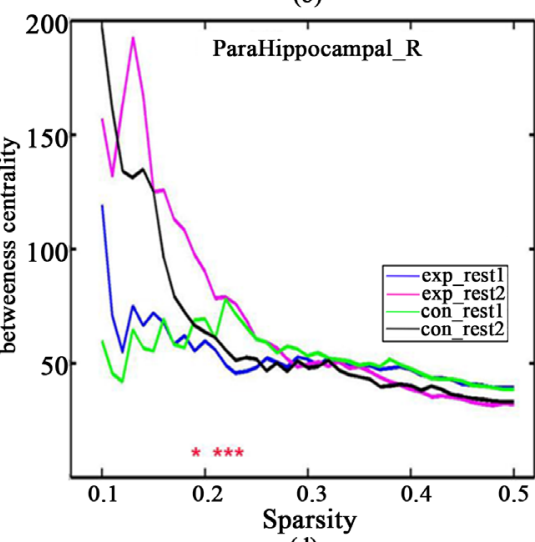

(d)

Figure 3. The variation of the nodal parameters that show significant interaction effect with the sparsity threshold for PCC.L/R and PHG.R during the pre-training and post-training resting runs for both groups. (a) Changes of betweenness centrality of PCC.L; (b) Changes of efficiency of PCC.L; (c) Changes of betweenness centrality of PCC.R; (d) Changes of betweenness centrality of PHG.R. Red asterisks indicate the significant difference between the pre-training and post-training resting runs of the experimental group $\left({ }^{*} \mathrm{FDR}\right.$-corrected $\left.p<0.05\right)$.

of the parameters that showed a significant interaction effect with the sparsity level. For the post-training versus the pre-training resting run, the experimental group showed a significant decrease in the degree, nodal efficiency and betweenness centrality of the SFGmed. L/R (see Figures 2(a)-(f)), in the betweenness centrality of the PCC.L, and in the nodal efficiency and betweenness centrality of the PCC.R (see Figures 3(a)-(c)) within some ranges of sparsity levels (FDR-corrected $p<0.05)$. Moreover, the experimental group produced significantly higher betweenness centrality of the PHG.R (see Figure 3(d)) for the post-training than the pre-training resting run within some ranges of sparsity levels (FDR-corrected $p<0.05$ ). The control group did not show any significant changes in DMN nodal graph properties after the training runs.

Importantly, we further divided the degree of each DMN node into two parts, including the intra-DMN degree and extra-DMN degree. The intra-DMN degree of a DMN node is defined as the number of edges that connect the node with the other nodes within the DMN. The extra-DMN degree of a DMN node is defined 
as the number of edges that connect the node with the other nodes outside of the DMN. A two-way repeated-measures ANOVA using group as a between-subject factor and state as a repeated-measures factor revealed that the intra-DMN degree of the SFGmed. L/R, the extra-DMN degree of the PCC.R and the extra-DMN degree of the PHG.R displayed significant interaction effect within some ranges of sparsity levels $(p<0.05)$. The variations of the intra-DMN degree of the SFGmed. L/R, the extra-DMN degree of the PCC.R and PHG.R with the sparsity level are presented in Figure 4. The simple effect analysis further revealed that the intra-DMN degree of the SFGmed. L/R and PHG.R and the extra-DMN degree of the PCC.R were significantly lower, and the extra-DMN degree of the PHG.R was significantly higher in the post-training resting run of the experimental group than in the pre-training resting run (FDR-corrected $p<$ 0.05). The DMN nodes of the control group did not produce any significant changes in the intra-DMN and extra-DMN degree.

\subsection{Results of the Global DMN Topography}

For each functional connectivity, a two-way repeated-measures ANOVA using group (experimental group and control group) as a between-subject factor and state (pre-training and post-training) as a repeated-measures factor was conducted in SPSS 20.0. If any functional connectivity showed a significant interac-
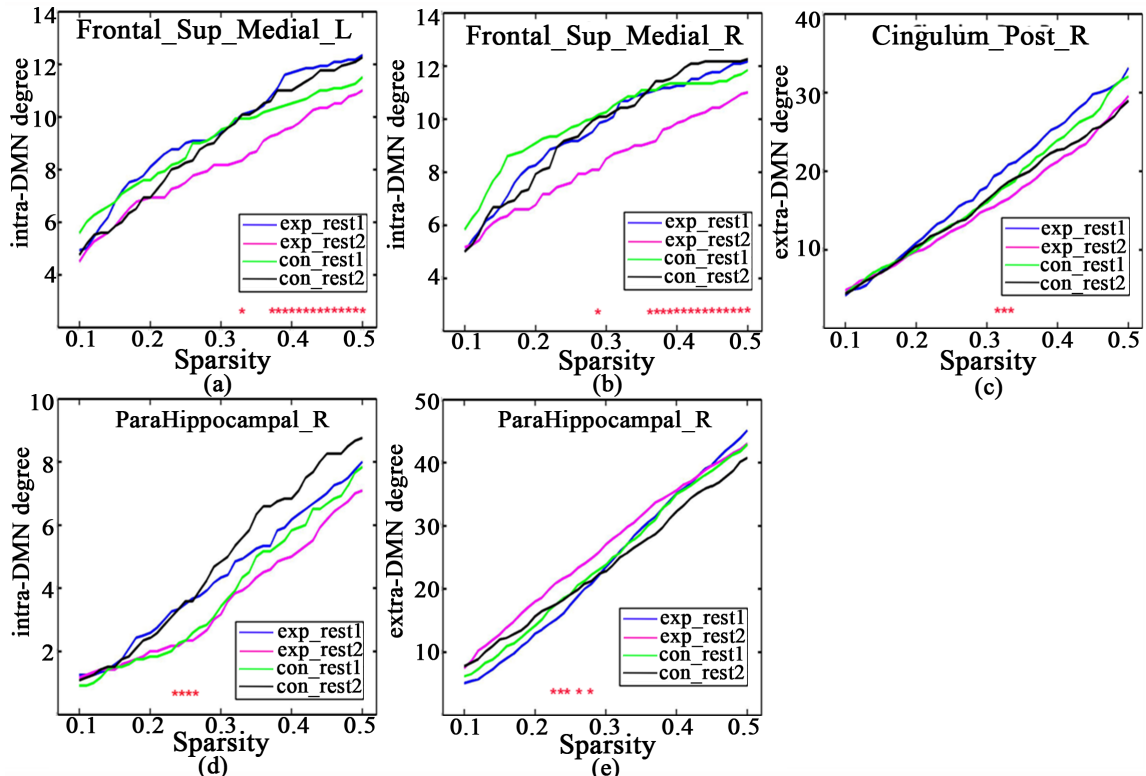

(c)

Figure 4. The variation of intra-DMN and extra-DMN degree with the sparsity level for the nodes that show significant interaction effect during the pre-training and post-training resting runs for both groups. (a) Changes of intra-DMN degree of the SFGmed. L; (b) Changes of intra-DMN degree of the SFGmed. R; (c) Changes of the extra-DMN degree of the PCC. R (d) Changes of intra-DMN degree of the PHG.R; (e) Changes of extraDMN degree of the PHG.R. Red asterisks indicate the significant difference between the pre-training and post-training resting runs of the experimental group $\left({ }^{*}\right.$ FDR-corrected $p$ $<0.05)$. 
tion effect $(p<0.05)$, tests of simple effect were further carried out and were corrected by the false discovery rate (FDR) method to examine the differences between the pre-training and post-training resting states in each group. The differences of the global DMN topography between the two resting runs of each group are shown in Figure 5. The functional connectivity that showed a significant interaction between the state and the group are colored in Figure 5. For the post-training versus pre-training run, the intra-DMN functional connectivity between the SFGmed. L and PHG.L, connectivity between the SFGmed. R and PHG.L and connectivity between the SFGmed. R and PHG.R were significantly decreased in the experimental group (see Figure 5(a)). For the extra-DMN functional connectivity, significant changes of the experimental group mainly occurred in the connectivity between the SFGmed/ Anterior cingulate/PHG/Angular and the regions outside of the DMN. In contrast, the control group did not show significant alteration of the intra-DMN functional connectivity and showed some significant alterations of the extra-DMN functional connectivity.

\section{Discussion}

In the present study, we utilized the rtfMRI technique and graph theory analysis method to investigate neurofeedback training-related changes in the topological

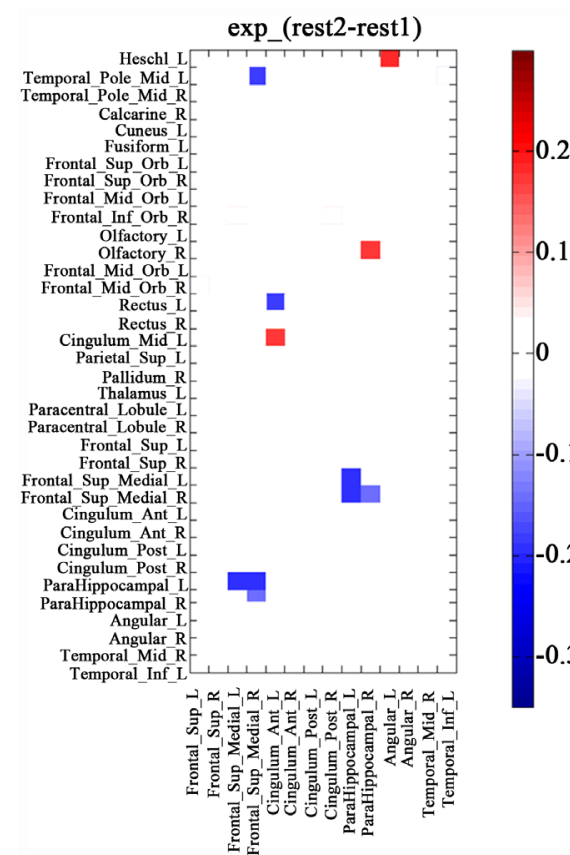

(a)

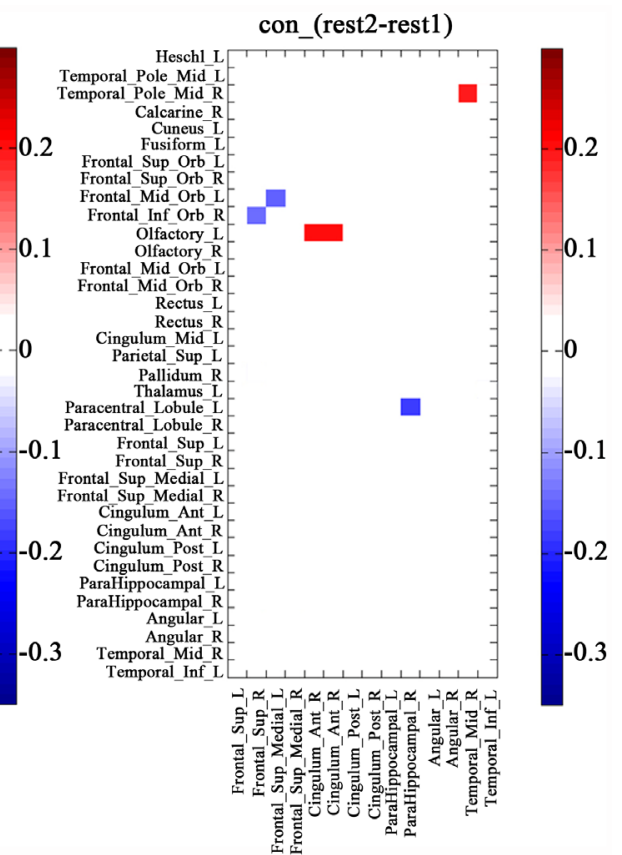

(b)

Figure 5. The variation maps of the global DMN topography. (a) Changes of functional connectivity for the post-training relative to the pre-training of the experimental group; (b) Changes of functional connectivity for the post-training relative to the pre-training of the control group. Color cells indicate the individual significant differences of functional connectivity between the post-training and pre-training resting runs for both groups. (FDR corrected, $p<0.05$ ). Warm color cells represent positive values while cold color cells represent negative values. 
properties of brain functional networks of the resting state. The main findings are as follows: 1) The brain network exhibited prominent small-world properties that cannot be changed by neurofeedback training; 2) The down-regulation of the PCC significantly reduced the functional connectivity between the SFGmed and PHG of the experimental group; and 3) the down-regulation of the PCC resulted in significant reductions in the nodal parameters of the SFGmed and PCC and significant increases in the nodal parameters of the PHG.

\subsection{Small-Worldness of the Whole Brain Network}

In this study, the whole brain network showed small-world properties during the pre-training and post-training resting run (see Figure 1), which is consistent with previous studies' findings that the human brain networks are small world (for reviews, see [31] [32]). The small-world mode indicates that the architecture of the networks contains dense local clustering between neighboring nodes and a short path length between any (distant) pair of nodes that have relatively few long-range connections [32]. The unchanged small-world properties after neurofeedback training further suggest that the functional organization of the brain maintained stable, robust and efficient small-world attributes for its internal complicated information processing. Moreover, the small-world organization also reflects an optimal balance between the global integration and local specialization of parallel information processing [32] [33]. Our results indicated that real-time training could not break the balance between the local specialization and global integration.

\subsection{Training-Related Alterations of the Nodal Graph Properties}

Our previous study demonstrated that most subjects in the experimental group can successfully reduce the activity of the PCC [15]. In this study, we found that the experimental group showed significant reductions in the betweenness centrality and efficiency of the PCC after the down-regulation of the PCC, while the control group did not show any significant changes in the PCC (see Figure 3(a) and Figure 3(b)). Moreover, the extra-DMN degree of the PCC of the experimental group was significantly reduced, and the intra-DMN degree did not change after neurofeedback training (see Figure 4(c)). The results indicated that the connectivity of the PCC with the brain nodes outside of the DMN after the down-regulation of the PCC was mainly reduced, which may lead to a reduction in information transfer from the PCC with the nodes outside of the DMN. Therefore, the betweenness centrality and efficiency of the PCC were reduced after the down-regulation of the PCC. The unchanged degree of the PCC in the intra-DMN of the experimental group further suggests that the PCC is an important key hub of the DMN [26] and maintains a more stable connectivity and information transfer with the nodes in the DMN than the nodes outside of the DMN, even if the activity of the PCC is down-regulated.

Compared with the pre-training resting run, the degree, nodal efficiency and betweenness centrality of the SFGmed.R and SFGmed. L of the experimental 
group were significantly decreased during the post-training resting run (see Figures 2(a)-(f)). Moreover, the experimental group significantly reduced the intra-DMN rather than the extra-DMN degree of the SFGmed. $\mathrm{R}$ and SFGmed. L (see Figure 4(a) and Figure 4(b)). The results showed that the down-regulation of the PCC largely reduced the information transmission ability of the SFGmed and the influence of the SFGmed over information flow between other nodes. Because the intra-DMN degree of the SFGmed and the functional connectivity between the SFGmed and PHG were significantly decreased after the neurofeedback training (see Figure 4(a) and Figure 4(b) and Figure 5(a)), it could be inferred that the SFGmed of the experimental group possibly reduced the information transmission with the PHG in the DMN. The PHG.R of the experimental group showed a significant increase in the betweenness centrality and the extra-DMN degree and a significant decrease in the intra-DMN degree after the neurofeedback training (see Figure 3(d), Figure 4(d) and Figure 4(e)). Moreover, the PHG.R of the experimental group showed significantly decreased connectivity with the SFGmed. $\mathrm{R}$ in the DMN and increased connectivity with regions outside of the DMN (see Figure 5(a)). The results could indicate that the real-time neurofeedback training increased the interaction between the PHG.R and the regions outside of the DMN, while it decreased the information transmission between the PHG.R and the regions within the DMN, especially the SFGmed. R.

Notably, several nodal graph properties of the SFGmed were significantly decreased, while the betweenness centrality of the PHG.R was significantly increased in the experimental group after the down-regulation of the PCC (see Figures 2(a)-(f), Figure 3(d)). The nodal graph property of the PHG.R seemed to show the opposite pattern compared to the SFGmed for the experimental group. Given that SFGmed covers pre-supplementary motor area (pre-SMA), the functional connectivity between the pre-SMA and PHG of the experiment group was significantly reduced after neurofeedback training because they were actively trying to downregulate PCC (while watching the PCC ROI signal activity) during training by performing a complex motor task [34]. It is possible that the memory processing and motor planning components get decoupled due to the complex motor task being visually guided in the case of the experimental group.As a result, it may lead to the reduced functional connectivity between the SFGmed and PHG, the reduced nodal graph properties of the SFGmed and the increased nodal graph property of the PHG.R after neurofeedback training. Our previous study also found that the down-regulation of the PCC resulted in the reduced activity of the SFGmed in the experimental group. The previous result is consistent with this study and further supports the inference that the levels of congruency of the down-regulation of the PCC with prior knowledge were reduced after neurofeedback training for the experimental group.

\subsection{Robustness and Stability of the Global Network Parameters}

No significant real-time training-related alterations were found in the global pa- 
rameters of the brain functional network for both the experimental group and control group. The results indicated that the real-time neurofeedback training could not change the global properties of brain networks in the resting state. For the functional network of the brain, the global properties are more stable and robust than the regional nodal properties. This finding is consistent with previous brain functional network studies [35] [36] that demonstrated that the intrinsic organization of the brain remained consistent during the resting states regardless of preceding experiences. Moreover, this finding also suggests that examining the intrinsic organization of the brain may provide additional insight into the dynamics of the brain that are induced by training.

\subsection{Limitations}

It should be noted that there are some limitations in this study. Firstly, this study did not include the control groups using neurofeedback from different brain regions as well as sham feedback. Thus, the differences of these two groups may come from not only neurofeedback but also working load and sensory input etc. Secondly, this study parceled each subject's data into 90 cortical and subcortical regions using the AAL atlas to construct the brain network. Some other atlases can be used to parcel each subject's data into more regions in future study.

\section{Conclusion}

To summarize, we investigated the impact of the down-regulation of the PCC on the topological properties of the brain functional network in the resting state using a graph theory analysis method. We observed that both the small-world properties of the brain functional network and their global network parameters remained stable and robust after the neurofeedback training. Moreover, the results indicated that the down-regulation of the core hub (PCC) in the DMN possibly reduced the information transfer of the PCC with the nodes outside of the DMN and reduced the functional connectivity between the SFGmed and PCC.

\section{Acknowledgements}

The authors are also grateful to Dr. Zhijiang Wang who kindly discussed some issues on graph theory method with us. This work is supported by Key Program of National Natural Science Foundation of China (61731003) and the National Natural Science Foundation of China (61671067).

\section{References}

[1] Ogawa, S. and Sung, Y.W. (2007) Functional Magnetic Resonance Imaging. Scholarpedia, 2, 3105. https://doi.org/10.4249/scholarpedia.3105

[2] Buckner, R.L. and Vincent, J.L. (2007) Unrest at Rest: Default Activity and Spontaneous Network Correlations. Neuroimage, 37, 1091-1096.

https://doi.org/10.1016/j.neuroimage.2007.01.010

[3] Lee, M.H., Smyser, C.D. and Shimony, J.S. (2013) Resting-State fMRI: A Review of Methods and Clinical Applications. American Journal of Neuroradiology, 34, 
1866-1872. https://doi.org/10.3174/ajnr.A3263

[4] Rosazza, C. and Minati, L. (2011) Resting-State Brain Networks: Literature Review and Clinical Applications. Neurological Sciences, 32, 773-785.

https://doi.org/10.1007/s10072-011-0636-y

[5] Ferreira, L.K. and Busatto, G.F. (2013) Resting-State Functional Connectivity in Normal Brain Aging. Neuroscience \&Biobehavioral Reviews, 37, 384-400. https://doi.org/10.1016/j.neubiorev.2013.01.017

[6] Greicius, M.D., Flores, B.H., Menon, V., Glover, G.H., Solvason, H.B., Kenna, H. and Schatzberg, A.F. (2007) Resting-State Functional Connectivity in Major Depression: Abnormally Increased Contributions from Subgenual Cingulate Cortex and Thalamus. Biological Psychiatry, 62, 429-437.

https://doi.org/10.1016/j.biopsych.2006.09.020

[7] Jiang, T., He, Y., Zang, Y. and Weng, X. (2004) Modulation of Functional Connectivity during the Resting State and the Motor Task. Human Brain Mapping, 22, 63-71. https://doi.org/10.1002/hbm.20012

[8] Tung, K.C., Uh, J., Mao, D., Xu, F., Xiao, G. and Lu, H. (2013) Alterations in Resting Functional Connectivity Due to Recent Motor Task. Neuroimage, 78, 316-324. https://doi.org/10.1016/j.neuroimage.2013.04.006

[9] Caria, A., Sitaram, R. and Birbaumer, N. (2012) Real-Time fMRI: A Tool for Local Brain Regulation. The Neuroscientist, 18, 487-501.

https://doi.org/10.1177/1073858411407205

[10] Emmert, K., Kopel, R., Sulzer, J., Brühl, A.B., Berman, B.D., Linden, D.E., et al. (2016) Meta-Analysis of Real-Time fMRI Neurofeedback Studies Using Individual Participant Data: How Is Brain Regulation Mediated? NeuroImage, 124, 806-812. https://doi.org/10.1016/j.neuroimage.2015.09.042

[11] Hampson, M., Scheinost, D., Qiu, M., Bhawnani, J., Lacadie, C. and Leckman, J.F. (2011) Biofeedback from the Supplementary Motor Area Reduces Functional Connectivity to Subcortical Regions. Brain Connectivity, 1, 91-98.

https://doi.org/10.1089/brain.2011.0002

[12] Scheinost, D., Stoica, T., Saksa, J., Papademetris, X., Constable, R.T., Pittenger, C. and Hampson, M. (2013) Orbitofrontal Cortex Neurofeedback Produces Lasting Changes in Contamination Anxiety and Resting-State Connectivity. Translational psychiatry, 3, e250. https://doi.org/10.1038/tp.2013.24

[13] Yuan, H., Young, K.D., Phillips, R., Zotev, V., Misaki, M. and Bodurka, J. (2014) Resting-State Functional Connectivity Modulation and Sustained Changes after Real-Time Functional Magnetic Resonance Imaging Neurofeedback Training in Depression. Brain connectivity, 4, 690-701. https://doi.org/10.1089/brain.2014.0262

[14] Megumi, F., Yamashita, A., Kawato, M. and Imamizu, H. (2015) Functional MRI Neurofeedback Training on Connectivity between Two Regions Induces Long-Lasting Changes in Intrinsic Functional Network. Frontiers in human neuroscience, 9, 160. https://doi.org/10.3389/fnhum.2015.00160

[15] Zhang, G., Zhang, H., Li, X., Zhao, X., Yao, L. and Long, Z. (2013) Functional Alteration of the DMN by Learned Regulation of the PCC Using Real-Time fMRI. IEEE Transactions on Neural Systems and Rehabilitation Engineering, 21, 595-606. https://doi.org/10.1109/TNSRE.2012.2221480

[16] Wang, J., Zuo, X. and He, Y. (2010) Graph-Based Network Analysis of Resting-State Functional MRI. Frontiers in Systems Neuroscience, 4, 16. https://doi.org/10.3389/fnsys.2010.00016 
[17] Zhang, H., Xu, L., Wang, S., Xie, B., Guo, J., Long, Z. and Yao, L. (2011) Behavioral Improvements and Brain Functional Alterations by Motor Imagery Training. Brain Research, 1407, 38-46. https://doi.org/10.1016/j.brainres.2011.06.038

[18] Jenkins, G. M. and Watts, D. G. (1968) Spectral Analysis and Its Applications Holden-Day. San Francisco, 243-238.

[19] Wang, L., Zhu, C., He, Y., Zang, Y., Cao, Q., Zhang, H., et al. (2009) Altered Small-World Brain Functional Networks in Children with Attention-Deficit/ Hyperactivity Disorder. Human Brain Mapping, 30, 638-649. https://doi.org/10.1002/hbm.20530

[20] Bullmore, E. and Sporns, O. (2009) Complex BRAIN NETWORKS: GRAPH Theoretical Analysis of Structural and Functional Systems. Nature Reviews Neuroscience, 10, 186-198. https://doi.org/10.1038/nrn2575

[21] Newman, M.E. (2003) The Structure and Function of Complex Networks. SIAM Review, 45, 167-256. https://doi.org/10.1137/S003614450342480

[22] Latora, V. and Marchiori, M. (2001) Efficient Behavior of Small-World Networks. Physical Review Letters, 87, Article ID: 198701. https://doi.org/10.1103/PhysRevLett.87.198701

[23] Watts, D.J. and Strogatz, S.H. (1998) Collective Dynamics of "Small-World" Networks. Nature, 393, 440-442.

[24] Maslov, S. and Sneppen, K. (2002) Specificity and Stability in Topology of Protein Networks. Science, 296, 910-913. https://doi.org/10.1126/science.1065103

[25] Humphries, M.D., Gurney, K. and Prescott, T.J. (2006) The Brainstem Reticular Formation Is a Small-World, Not Scale-Free, Network. Proceedings of the Royal Society of London B: Biological Sciences, 273, 503-511. https://doi.org/10.1098/rspb.2005.3354

[26] Fransson, P. and Marrelec, G. (2008) The Precuneus/Posterior Cingulate Cortex Plays a Pivotal Role in the Default Mode Network: Evidence from a Partial Correlation Network Analysis. Neuroimage, 42, 1178-1184. https://doi.org/10.1016/j.neuroimage.2008.05.059

[27] Achard, S. and Bullmore, E. (2007) Efficiency and Cost of Economical Brain Functional Networks. PLOS Computational Biology, 3, e17. https://doi.org/10.1371/journal.pcbi.0030017

[28] Freeman, L.C. (1977) A Set of Measures of Centrality Based on Betweenness. Sociometry, 35-41. https://doi.org/10.2307/3033543

[29] Fox, M.D., Snyder, A.Z., Vincent, J.L., Corbetta, M., Van Essen, D.C. and Raichle, M.E. (2005) The Human Brain Is Intrinsically Organized into Dynamic, Anticorrelated Functional Networks. Proceedings of the National Academy of Sciences of the United States of America, 102, 9673-9678. https://doi.org/10.1073/pnas.0504136102

[30] Benjamini, Y. and Hochberg, Y. (1995) Controlling the False Discovery Rate: A Practical and Powerful Approach to Multiple Testing. Journal of the Royal Statistical Society. Series B (Methodological), 289-300.

[31] Stam, C.J. and Reijneveld, J.C. (2007) Graph Theoretical Analysis of Complex Networks in the Brain. Nonlinear Biomedical Physics, 1, 3. https://doi.org/10.1186/1753-4631-1-3

[32] Bassett, D.S. and Bullmore, E.D. (2006) Small-World Brain Networks. The Neuroscientist, 12, 512-523. https://doi.org/10.1177/1073858406293182

[33] Sporns, O. and Zwi, J.D. (2004) The Small World of the Cerebral Cortex. Neuroin- 
formatics, 2, 145-162. https://doi.org/10.1385/NI:2:2:145

[34] Kim, J.H., Lee, J.M., Jo, H.J., Kim, S.H., Lee, J.H., Kim, S.T. and Saad, Z.S. (2010) Defining Functional SMA and Pre-SMA Subregions in Human MFC using Resting State fMRI: Functional Connectivity-Based Parcellation Method. Neuroimage, 49, 2375-2386. https://doi.org/10.1016/j.neuroimage.2009.10.016

[35] Wang, Z., Liu, J., Zhong, N., Qin, Y., Zhou, H. and Li, K. (2012) Changes in the Brain Intrinsic Organization in Both On-Task State and Post-Task Resting State. Neuroimage, 62, 394-407. https://doi.org/10.1016/j.neuroimage.2012.04.051

[36] Rzucidlo, J.K., Roseman, P.L., Laurienti, P.J. and Dagenbach, D. (2013) Stability of Whole Brain and Regional Network Topology within and between Resting and Cognitive States. PLOS ONE, 8, e70275.

https://doi.org/10.1371/journal.pone.0070275 